\title{
Large Scale Infrared-Based Remote Sensing of Turbulence Metrics in Surface Waters: Going Beyond Mean Flow
}

\author{
Seth A. Schweitzer ${ }^{1 *}$, Edwin A. Cowen ${ }^{1}$ \\ ${ }^{1}$ DeFrees Hydraulics Laboratory, School of Civil \& Environmental Engineering, Cornell University, \\ Ithaca, NY* seth.schweitzer@cornell.edu
}

\begin{abstract}
In recent years field-scale applications of image-based velocimetry methods, often referred to as large scale particle image velocimetry (LSPIV), have been increasingly deployed. These velocimetry measurements have several advantages - they allow high resolution, non-contact measurement of surface velocity over a large two dimensional area, from which the bulk flow can be inferred. However, visiblelight LSPIV methods can have significant limitations. The water surface often lacks natural features that can be tracked in the visible and generally requires seeding with tracer particles, which creates concerns regarding the fidelity with which tracer particles track the flow, and introduces challenges in achieving sufficient and uniform seeding density, in particular in regions with appreciable velocity accelerations such as turbulence. In LSPIV, image collection is generally limited to daylight hours, and can suffer from non-uniformity of illumination across the camera's field of view. Due to these issues LSPIV often requires spatio-temporal averaging, and as a result is generally able to extracting the mean, but not the instantaneous, velocity field, and hence is often not a suitable tool for calculating turbulence metrics of the flow.
\end{abstract}

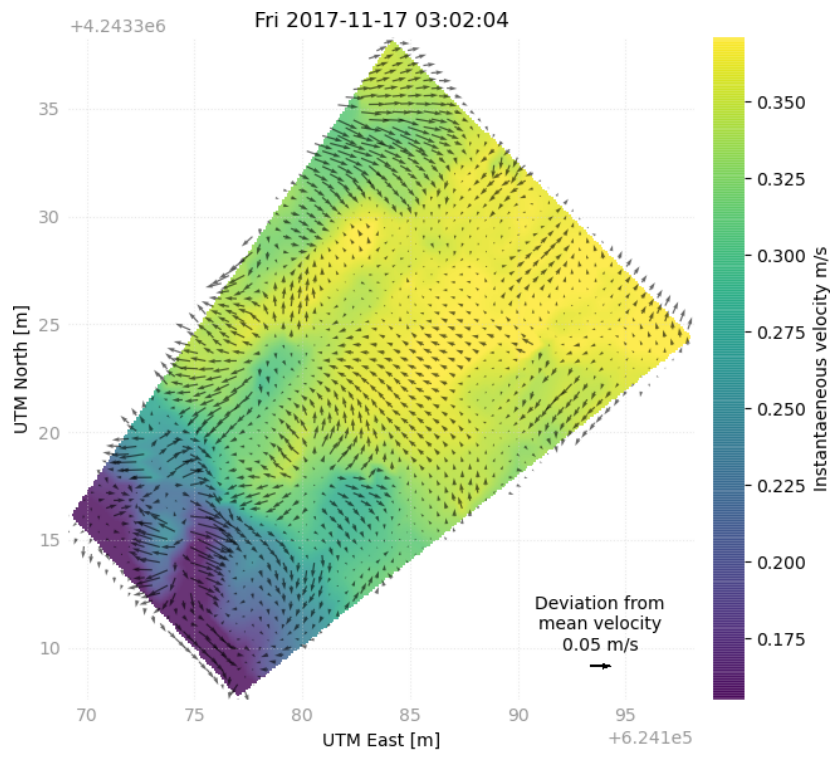

Figure 1: Instantaneous velocity field measured by IR-QIV over several hundred $\mathrm{m}^{2}$ at the surface of a river. Background color indicates the local instantaneous velocity. Arrows indicate the perturbation from the mean velocity, i.e., $\vec{u}^{\prime}$, the difference between the local instantaneous and mean velocity.
We present an alternative to visiblelight LSPIV that avoids these seeding and illumination issues: infrared quantitative image velocimetry (IR-QIV), which uses infrared (IR) images to accurately capture temperature patterns at the water surface with high thermal and spatial resolution (Schweitzer and Cowen, under review). Motion of the patterns is tracked over time, from which the surface velocity field is calculated (figure 1). In natural flows small temperature differences are created in the surface skin due to spatial heterogeneity in turbulent stirring and heat exchange between air and water. These spatial differences in temperature form a rich texture of patterns on the water surface that are observable in IR images (figure 2). IR images are a record of radiation emitted by the water surface, with no external illumination, and no concerns of possible differences between motion of tracer particles and the flow. In contrast to LSPIV, IR-QIV is able to extract the instantaneous velocity field reliably and robustly. These measurements can be used for a range of applications, including interaction of fish with structures and flow features, and non-contact estimation of bed stress, bathymetry and discharge. In this presentation we will provide an overview of our developed IR-QIV technique and 
detail the environmental conditions required for high quality IR image collection, and dealing with images collected under sub-optimal environmental conditions. These issues are key for optimizing IR-QIV for accurate turbulence measurements.

We use the minimum quadratic difference (MQD) method for pattern matching (Gui and Merzkirch, 1996, 2000), as it is more suitable than cross-correlation for images containing gradients of intensity and not discrete particles (Cowen et al., 2010). This method generates, at each interrogation location, a surface $D(m, n)=\frac{1}{M N} \sum_{i=1}^{M} \sum_{j=1}^{N}\left(g(i, j)-g^{\prime}(i+m, j+n)\right)^{2}$, where $g(i, j)$ represents a subwindow of dimensions $M, N$ with a corner at pixel-coordinates $(i, j)$, and $g^{\prime}(i+m, j+n)$ represents a similar subwindow from a subsequent image, displaced by $(m, n)$ pixels. Lower values of the surface $D$ indicate a greater similarity (smaller quadratic difference) between the pattern of pixel intensities in the two images at that displacement. We use a method that considers image entropy to optimize parameters such as the subwindow dimensions $M, N$, and the temporal separation $\Delta t$ between images.
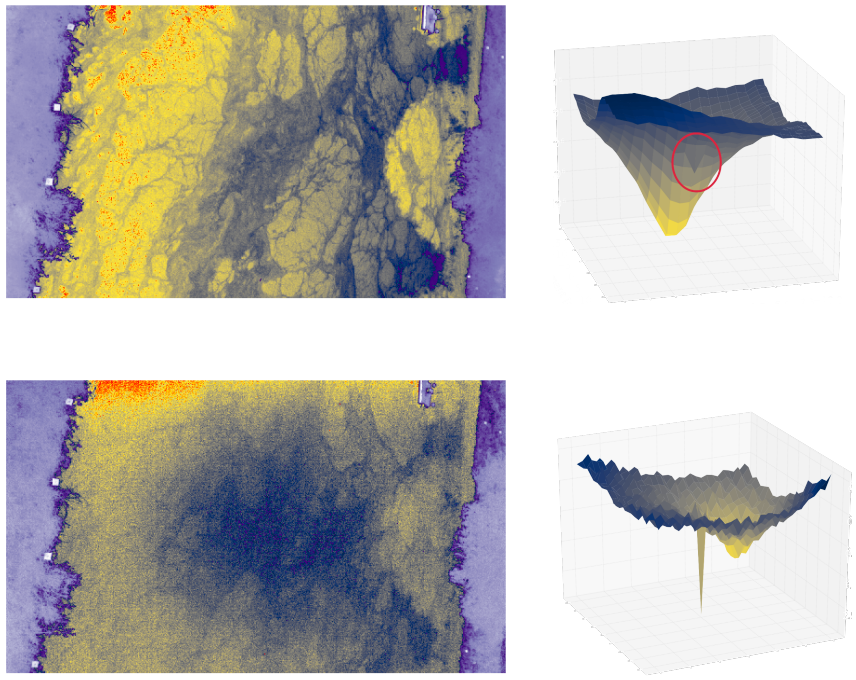

Figure 2: IR image of a river surface under environmental conditions leading to high (left, top), and low (left, bottom) dynamic range image, and representative surface $D$ for a single location in each of the images (right). In both examples there a local minimum at the coordinate corresponding to zero displacement (marked with a red circle).

The dynamic range of IR images is controlled by the range of temperature differences at the water surface, as well as the camera's sensitivity and position. In the measurement described in this presentation, IR images exhibited high dynamic range when the difference between bulk water temperature and wet-bulb air temperature was greater than $\sim 3{ }^{\circ} \mathrm{C}$. Smaller air-water temperature differences (corrected for latent heat) lead to low dynamic range in the image, and as a result to a large number of zero velocity results. The environmental conditions required for sufficient image quality to avoid zero-displacement issues will vary between measurements, depending on factors including the camera's sensitivity and internal noise level, optical setup (e.g., viewing angle, distance, and optics), and environmental conditions such as wind velocity and turbulence intensity of the flow.

\section{Acknowledgements}

Funding for this work was provided by the California Department of Water Resources (DWR). 


\section{References}

Cowen EA, Dudley RD, Liao Q, Variano EA, and Liu PLF (2010) An insitu borescopic quantitative imaging profiler for the measurement of high concentration sediment velocity. Experiments in Fluids 49:77-88

Gui L and Merzkirch W (1996) A method of tracking ensembles of particle images. Experiments In Fluids 21:465-468

Gui L and Merzkirch W (2000) A comparative study of the mqd method and several correlation-based piv evaluation algorithms. Experiments in Fluids 28:36-44

Schweitzer SA and Cowen EA (under review) Instantaneous river-wide water surface velocity field measurements at centimeter scales using infrared quantitative image velocimetry. Water Resources Research 